\title{
Community Obstetrical Units Less Likely than Academic Units to Have Universal COVID-19 Testing
} \author{
Mary E. Norton, $\mathrm{MD}^{6}$ Sindhu K. Srinivas, MD, MSCE ${ }^{7}$ \\ ${ }^{1}$ Department of Obstetrics and Gynecology, Brown University Warren \\ Alpert Medical School, Providence, Rhode Island \\ 2 Department of Obstetrics and Gynecology, University of South \\ Florida, Tampa, Florida \\ ${ }^{3}$ Department of Obstetrics and Gynecology, Duke University School \\ of Medicine, Durham, North Carolina \\ ${ }^{4}$ UCLA School of Medicine, Los Angeles, California \\ ${ }^{5}$ Center for Fetal Medicine and Women's Ultrasound, Los Angeles, \\ California \\ ${ }^{6}$ Department of Obstetrics, Gynecology and Reproductive Sciences, \\ University of California, San Francisco, San Francisco, California \\ ${ }^{7}$ Department of Obstetrics and Gynecology, Maternal and Child \\ Health Research Center, Perelman School of Medicine, University of \\ Pennsylvania, Philadelphia, Pennsylvania
}

Erika F. Werner, MD ${ }^{1}$ Judette M. Louis, MD² Brenna Hughes, MD ${ }^{3}$ Christina Shih-chi Han, MD ${ }^{4,5}$

Am J Perinatol 2020;37:1074-1076.

Current evidence from a high-prevalence area suggests that many pregnant women with novel coronavirus disease 2019 (COVID-19) are asymptomatic when they present for delivery. ${ }^{1}$ Obstetric deliveries occur at both academic and community hospitals and the site of delivery is not likely to be altered by the pandemic. If COVID-19 specific resources differ between academic and community hospital obstetric units, this could result in disparate disease transmission rates and care. This has the potential to place large numbers of patients, their families, and health care workers at risk. In response to member concerns, the Society for Maternal-Fetal Medicine (SMFM) surveyed its state liaison representatives using a web-based survey (https://www.surveymonkey.com/r/COVIDSLNsurvey) regarding COVID-19 testing and access to personal protective equipment (PPE) on obstetrical units between April 7, 2020 and April 14, 2020. We sought to determine current COVID-19 testing strategies on admission and PPE use at delivery across the United States.

Of the 56 maternal-fetal medicine physicians who responded from 38 states and the District of Columbia, 63\% $(n=35)$ were from academic institutions with the remainder from community hospitals. The 1-week response rate was $16.6 \%$ (337 members received the survey) from $78 \%$ of the states with members. In this small but geographically diverse sample, academic institutions and community hospitals had a similar volume of COVID-19 obstetric patients admitted per

received

April 28, 2020

accepted after revision

April 29, 2020

published online

May 19, 2020
Address for correspondence Erika F. Werner, Department of Obstetrics and Gynecology, Brown University Warren Alpert Medical School, 101 Dudley Street, Providence, RI 02906 (e-mail: EWerner@kentri.org).

week ( - Fig. 1, $p=0.76)$. Among respondents, 20\% (11/56) reported that their hospitals were performing universal testing for women admitted for inpatient obstetrical care and 27\% $(15 / 56)$ were considering but had not initiated this practice. Universal testing varied within states and did not correlate with COVID-19 inpatient obstetrical volumes; 31\% (5/16) of hospitals that admitted three or more COVID-19 affected patients per week were performing universal testing compared with $15 \%(6 / 40)$ of hospital that admitted less than three COID-19 affected patients per week, $p=0.26$. Universal testing rates differed significantly between hospitals types with $29 \%$ (10/35) of academic-affiliated respondents reporting universal testing compared with $5 \%(1 / 21)$ of community hospital $(p=0.04)$. All respondents reported adequate access to surgical masks, 96\% (54/56) reported using N95s for deliveries of COVID-19 affected patients and 25\% (14/56) reported using N95s for all deliveries. Universal N95 masking at delivery did not differ significantly between academic and community hospitals (29\% compared with $19 \%, p=0.53$ ).

Unlike many gynecologic visits that can be postponed or conducted virtually, obstetrical delivery timing often cannot be adjusted. Therefore, it is critical to develop strategies to ensure the safety of patients and health care workers in this environment. Although practices and guideline are rapidly evolving, this recent survey suggests that academic and community hospitals are encountering COVID-19 patients and recommending

Copyright $\odot 2020$ by Thieme Medical Publishers, Inc., 333 Seventh Avenue, New York, NY 10001, USA.

DOI https://doi.org/ 10.1055/s-0040-1712454. ISSN 0735-1631. 


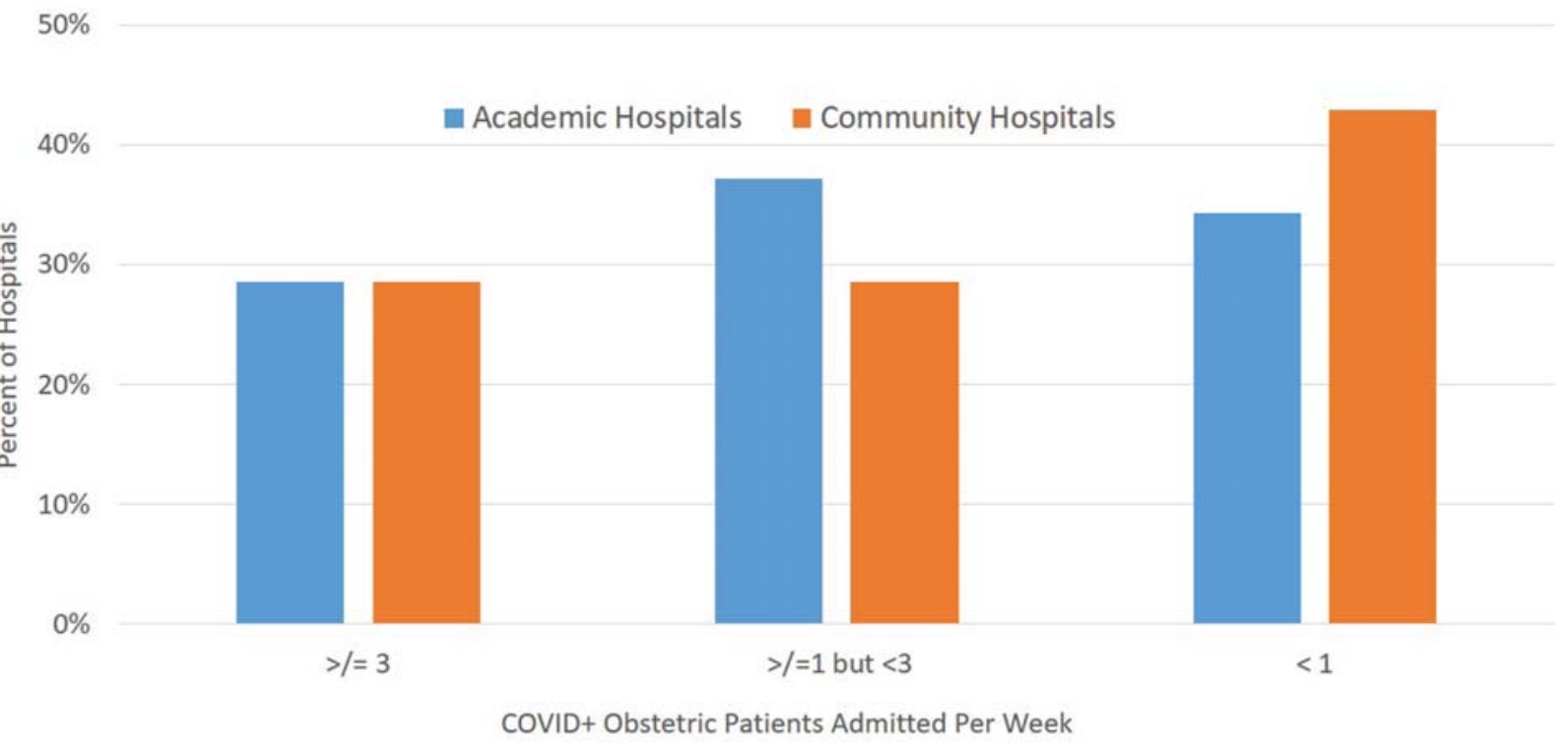

Fig. 1 COVID-19-positive obstetric patient volume by hospital affiliation. COVID-19, novel coronavirus disease 2019.

universal N95 masking at the time of delivery at similar rates. However, universal COVID-19 testing at the time of obstetrical admission is more common at academic than community hospitals and does not correlate with COVID-19-positive patient volume. These imbalances in testing practices may prevent universal COVID-19 testing from achieving its intent; identifying asymptomatic carriers across a community to mitigate disease spread. Low-prevalence areas can very quickly become high-prevalence areas. Therefore, implementation of universal testing should be strongly considered in all obstetric hospitals, regardless of their academic or community affiliation as an important strategy to reduce community spread.

Conflict of Interest

None declared.

\section{Reference}

1 Sutton D, Fuchs K, D’Alton M, Goffman D. Universal screening for SARS-CoV-2 in women admitted for delivery. N Engl J Med 2020. Doi: 10.1056/NEJMc2009316 